\title{
Lesiones y fútbol base: un análisis en dos clubes de la Región de Murcia Injuries and young football players: an analysis in two clubs of Murcia Region
}

\author{
*Aurelio Olmedilla Zafra, ** $\mathrm{M}^{\mathrm{a}}$ Dolores Andreu Álvarez, ***Francisco J. Ortín Montero y **Amador Blas Redondo \\ *Universidad Católica San Antonio (España), ** Sociedad Murciana de Psicología de la Actividad Física y el Deporte (España), \\ *** Universidad de Murcia (España)
}

Resumen: La literatura científica ha mostrado la importancia y el aumento que las lesiones han tenido en el fútbol en los últimos años. El objetivo del presente trabajo es describir las lesiones y determinar los factores más relevantes de éstas, sufridas por futbolistas jóvenes de dos clubes de la Región de Murcia. La muestra está formada por 72 futbolistas, de edades comprendidas entre los 14 y los 24 años, con una media de 17,2 años $( \pm 2,7)$. La información relativa a los datos deportivos y a las lesiones se recogió mediante un cuestionario de auto-informe. Los resultados muestran que las lesiones producidas durante los entrenamientos duplican a las producidas en partidos; y que las lesiones más frecuentes son musculares y de carácter leve o moderado. A la vista de los resultados se discute sobre la importancia de analizar, entre otros aspectos, los tipos de entrenamiento realizados por los equipos implicados, ya que pudiera ser un factor desencadenante de lesión.

Palabra clave: fútbol base, lesiones deportivas, entrenamiento, competición.

\begin{abstract}
The scientific literature has showed the importance and the increase that injuries have had in football in the last years. The aim of the present work is to describe the injuries and to determine the most relevant factors of them, suffered by young football players of two clubs of Murcia Region. The sample is composed by 72 football players, of ages between 14 and 24 years, with an average of 17,2 years $( \pm 2,7)$. The information relative to the sports data and injuries was gathered by means of an auto-report questionnaire. The results show that the injuries produced during trainings duplicate to those produced in matches; and that the most frequent injuries are muscular and of slight or moderate character. In view of the results it is discussed the importance of analyzing, among other aspects, the types of training realized by the implicated teams, since it could be a factor which causes injury. Key words: young football players, sports injuries, training, competition.
\end{abstract}

\section{Introducción}

El incremento paulatino de las lesiones en el fútbol ha hecho que los profesionales de la salud que trabajan en el ámbito deportivo manifiesten preocupación. Así por ejemplo, al inicio de la temporada 2006/07 el gran número de lesiones sufridas por futbolistas de la primeray segunda división española, hizo que la Asociación Española de Médicos de Equipos de Fútbol (AEMEF) junto a la Sociedad Española de Traumatología del Deporte(SETRADE, 2007) emitieran un comunicado manifestando su preocupación por este hecho, así como puesta en marcha de estudios de carácter epidemiológico que proporcionen las bases para debatir científicamente e intentar solucionar la problemática de la incidencia lesional.

Diferentes estudios epidemiológicos muestran la gran importancia que la lesión tiene en la práctica del fútbol (Hawkins y Fuller, 1999; Lüthje et al., 1996; Majewski, Sussanne y Klaus, 2006; Nicholl, Coleman y Williams, 1995; Nielsen e Yde, 1989). La mayoría de trabajos se han centrado en el fútbol profesional (Arnason et al., 2004; Hägglund, Walden y Ekstrand, 2003; Morgan y Oberlander, 2001); sin embargo, la mayor representatividad actual del fútbol femenino y del fútbol de formación ha hecho que el interés de los investigadores se dirija, también, hacia éstos (Barcala, Palacios, García y Oleagordia, 2007; Emery, Meeuwisse y Hartmann, 2005; Faude, Junge, Kindermann y Dvorak, 2005; Louw, Manilall y Grimmer, 2008; Malliou, Gioftsidou, Pafis, Beneka y Godolias, 2004; Östenberg y Roos, 2000; Söderman, Pietilä, Alfredson y Werner, 2002)

La incidencia de lesiones en el fútbol base es menor que en el profesional (Kibler, 1995), aunque se incrementa según aumenta la edad de los jugadores (Schmidt-Olsen, Jorgensen, Kaalund y Sorensen, 1991), por lo que resulta de gran interés conocer los datos que proporcionan los estudios con futbolistas profesionales. En este sentido, Hawkins, Hulse, Wilkinson, Hodson y Gibson (2001), analizan 6030 lesiones recogidas en 91 clubes ingleses de fútbol profesional, indicando una media de 1,3 lesiones por jugador y temporada, entre unos 24,2 y 40,2 días perdidos

Fecha recepción: 09-10-08 - Fecha envío revisores: 12-10-08 - Fecha de aceptación: 20-02-09 Correspondencia: Aurelio Olmedilla Zafra

Campus de los Jerónimos, s/n

30107 Guadalupe (Murcia)

E-mail: aolmedilla@pdi.ucam.edı de entrenamiento y el $78 \%$ de los futbolistas se pierden, al menos, un partido de competición.

En la revisión realizada por Junge y Dvorak (2004), las lesiones más frecuentes en fútbol son las de tobillo, de rodilla y de los músculos del tren inferior; específicamente esguinces, tendinitis y contusiones. La mayoría de las lesiones están causadas por traumatismos, contactos con otro jugador (Hawkins y Fuller, 1998, 1999), o por sobreentrenamiento (Arnason, Gudmumsson, Dahl y Johansson, 1996; Nielsen e Yde, 1989); además, en competiciones internacionales aumenta la proporción de lesiones (Fuller, Junge y Dvorak, 2004; Junge, Dvorak y Graf-Baumann, 2004). La mayoría de autores indican que las lesiones producidas por factores ajenos al contacto con otros se dan en una proporción entre el 26 y el 59\% del total de lesiones (Hawkins y Fuller, 1999; Lüthje et al., 1996) y éstas se producen, básicamente, durante la carrera o en cambios de dirección. Aproximadamente, entre un 20 y un $25 \%$ de las lesiones son recaídas del mismo tipo de lesión (Arnason, et al., 1996; Hawkins y Fuller, 1999), donde la historia de lesiones anteriores y una inadecuada recuperación de las mismas son factores de riesgo que incrementan su producción (Arnason et al., 2004; Dvorak et al., 2000; Hawkins et al., 2001).

El estudio de los factores de riesgo es una constante en las investigaciones centradas en el fútbol base, con criterios muy similares respecto al estudio del fútbol profesional, aunque siempre desde la perspectiva de la evolución psicosocial y deportiva de los jóvenes jugadores. En este sentido, para Hawkins y Fuller (1999) es muy importante controlar la participación de jóvenes futbolistas en la alta competición; en su estudio encontraron que el número de lesiones aumentaba significativamente durante la segunda mitad de la temporada, en contraposición de los jugadores profesionales (en los que disminuía). Aspectos como el partido o el entrenamiento, el tiempo de juego, los tipos de entrenamiento, la posición de juego, la edad y la categoría, y la historia de lesiones anteriores han sido variables que se han sumado a los estudios de los diferentes investigadores.

En general, las lesiones de los jóvenes futbolistas se producen en los partidos con una frecuencia mayor que en los entrenamientos (Emery et al., 2005; Faude et al., 2005), aunque el tipo de entrenamiento también parece ser un factor importante, dado que excesivas cargas de trabajo podrían aumentar el riesgo de lesión (Faude et al., 2005; Nielsen e Yde, 1989). En este sentido, los ejercicios de equilibrio propioceptivo podrían ayudar a prevenirlas (Gioftsidou y Malliou, 2006; Malliou et al., 
2004). Respecto al tiempo de juego, la mayoría de trabajos optan por contabilizar el número de lesiones por cada 1000 horas de competición, variando entre las 2,4 lesiones encontradas por Kibler (1995), las 3,7 por Schmidt-Olsen et al. (1991), las 5,6 por Emery et al. (2005), las 7,8 por Kontos (2000), las 11,7 por Maehlum et al. (1986), hasta las 19,1 por Schmidt-Olsen, Buenemann, Lade y Briscoe (1985) cuando en este caso se contabilizan las lesiones menores.

Los estudios respecto a la influencia de la edad y la categoría parecen indicar que a mayor edad mayor probabilidad de lesionarse del jugador (Majewski et al., 2006; Schmidt-Olsen et al., 1991) aunque en algunos trabajos (Emery et al., 2005) la mayor incidencia de lesiones se producía a la edad de 14 años, lo que en nuestro país se considera categoría cadete. La historia de lesiones anteriores del jugador es una variable que incrementa la probabilidad de lesionarse (Emery et al., 2005).

El objetivo del presente trabajo es describir las lesiones sufridas, atendiendo al momento de producirse la lesión, al número de lesiones, al tipo de lesión y a la gravedad de la misma, por los futbolistas de dos clubes de gran importancia y representatividad en el fútbol base de la Región de Murcia, y establecer si existen diferencias entre ellos.

\section{Método}

\subsection{Participantes}

La muestra está formada por 72 futbolistas, de edades comprendidas entre los 14 y los 24 años, con una media de 17,2 años (dt de 2,7). Todos los jugadores pertenecían a uno de los dos clubes con mayor tradición en el fútbol base regional (Ranero CF y Real Murcia SAD), en equipos que competían en la Federación de Fútbol de la Región de Murcia. En la Tabla 1 se pueden ver los datos descriptivos más relevantes de la muestra.

\begin{tabular}{|c|c|c|c|}
\hline \multicolumn{4}{|c|}{ Tabla 1. Características descriptivas de los jugadores } \\
\hline & & RANERO CF & R. MURCIA SAD \\
\hline \multirow{2}{*}{ Edad } & Intervalo & $14-18$ & $17-24$ \\
\hline & Media & 15,31 & 19,45 \\
\hline \multirow{2}{*}{ Entreno semanal (días) } & Intervalo & $3-3$ & $4-6$ \\
\hline & Media & 3 & 4,70 \\
\hline \multirow{2}{*}{ Entreno diario (min.) } & Intervalo & $90-120$ & $90-120$ \\
\hline & Media & 100 & 114 \\
\hline \multirow{2}{*}{ Partidos jugados } & Intervalo & $1-33$ & $2-25$ \\
\hline & Media & 19,12 & 12,9 \\
\hline \multirow{2}{*}{$\begin{array}{l}\text { Lesiones de jugadores } \\
\left(\mathrm{n}^{\circ} \text { de lesiones sufridas por cada }\right. \\
\text { jugador) }\end{array}$} & Intervalo & $0-3$ & $0-5$ \\
\hline & Media & 1,29 & 1,70 \\
\hline \multirow{3}{*}{$\begin{array}{l}\text { Categoría deportiva } \\
\text { ( } \mathrm{n}^{\circ} \text { de jugadores por categoría) }\end{array}$} & Cadete & $22(58 \%)$ & \\
\hline & Juvenil & $16(42 \%)$ & $20(60,6 \%)$ \\
\hline & $3^{a}$ División & & $13(39,4 \%)$ \\
\hline \multirow{4}{*}{ Puesto táctico habitual } & Portero & $3(7,7 \%)$ & $3(9,4 \%)$ \\
\hline & Defensa & $11(29,2 \%)$ & $9(28,3 \%)$ \\
\hline & Medio & $17(41,6 \%)$ & $14(43,4 \%)$ \\
\hline & Delantero & $7(18,5 \%)$ & $6(18,9 \%)$ \\
\hline
\end{tabular}

\subsection{Instrumentos y procedimiento}

A todos los futbolistas se les administró un cuestionario de autoinforme(Olmedilla, Ortega y Abenza, 2007) que recogía información de tipo deportivo, así como la derivada de las lesiones. La información solicitada respecto a las lesiones hacía referencia al hecho de si habían sufrido lesión o no durante la temporada en curso, el número de lesiones que habían sufrido, si éstas se habían producido en entrenamientos o en partidos, el tipo de lesión sufrida (muscular, contusión, esguince, tendinitis, lesión ósea), y su gravedad. Siguiendo las sugerencias de diversos autores (Buceta, 1996; Olmedilla, García-Montalvo y Martínez-Sánchez, 2006) en el cuestionario se describían cuatro niveles de gravedad de lesiones: lesión leve (requiere tratamiento e interrumpe al menos un día de entrenamiento), moderada (requiere tratamiento y obliga al futbolista a interrumpir seis días su participación en entrenamientos e, incluso, en algún partido), grave (supone de uno a tres meses de baja deportiva) y muy grave (supone cuatro meses o más de baja deportiva, requiere hospitalización, intervención quirúrgica y, a veces, precisa de rehabilitación constante para evitar empeoramiento).
Tras solicitar permiso a los responsables de los clubes objeto de estudio, se procedió a informar a los entrenadores de los equipos implicados. Por último, en el momento de administrar los cuestionarios a los jugadores, se informó a éstos del carácter anónimo de las respuestas, así como el tratamiento protocolario de los resultados de la investigación. Los cuestionarios fueron administrados, por psicólogos especialistas en psicología del deporte, en las instalaciones que los clubes disponen para los entrenamientos de sus equipos, durante el último mes de competición oficial.

\subsection{Análisis estadístico}

Se ha realizado un enfoque correlacional descriptivo, explorando la asociación entre las variables analizadas mediante pruebas $\langle\downarrow\rangle\rangle$ de Student para diferencias de medias independientes, análisis de correlación de Pearson y análisis ANOVA (con pruebas post-hoc «t» de Tukey). Los análisis estadísticos los realizamos con el programa SPSS 15.0

\section{Resultados}

En la Tabla 2 se puede observar la distribución del número de lesiones y el número de jugadores que las han sufrido. Se han registrado un total de 105 lesiones. La mayoría de los jugadores (un 57\%), o no se han lesionado (15,3\%), o solo han tenido 1 lesión (un 41,7\%). El porcentaje de jugadores que han sufrido 3 o más lesiones es pequeño $(15,3 \%)$, y resulta relativamente común sufrir dos lesiones $(26,4 \%)$.

\begin{tabular}{|c|c|c|c|}
\hline \multicolumn{2}{|c|}{$\mathrm{N}^{\mathrm{o}}$ de lesiones } & $\begin{array}{l}\mathrm{N}^{\circ} \text { de jugadores según el } \\
\text { número de lesiones sufridas }\end{array}$ & Porcentaje \\
\hline \multicolumn{2}{|c|}{0 lesiones sufridas } & 11 & 15,3 \\
\hline \multicolumn{2}{|c|}{1 lesiones sufridas } & 30 & 41,7 \\
\hline \multicolumn{2}{|c|}{2 lesiones sufridas } & 19 & 26,4 \\
\hline \multicolumn{2}{|c|}{3 lesiones sufridas } & 8 & 11,1 \\
\hline \multicolumn{2}{|c|}{4 lesiones sufridas } & 2 & 2,8 \\
\hline \multicolumn{2}{|c|}{5 lesiones sufridas } & 1 & 1,4 \\
\hline Total $n^{\circ}$ de lesiones & 105 & & 98,6 \\
\hline \multicolumn{2}{|l|}{ Perdido Sistema } & 1 & 1,4 \\
\hline \multicolumn{2}{|l|}{ Total Jugadores } & 72 & 100,0 \\
\hline
\end{tabular}

En la Tabla 3 se puede observar la distribución de las lesiones sufridas por los jugadores en función del lugar de ocurrencia, del tipo de lesión y de su gravedad. Destaca el hecho de que son mucho mas frecuentes las lesiones en los entrenamientos que en los partidos; así, el $56,5 \%$ de los jugadores no sufre ninguna lesión durante los partidos, mientras que solo un $33,3 \%$ no las sufre en los entrenamientos; además un $21,7 \%$ de los jugadores sufre dos lesiones en los entrenamientos, mientras únicamente un 4,3\% les sucede en los partidos. En cuanto al tipo de lesión, la mayoría son musculares (55), seguidas de esquinces (18), y sufridas por un $54,9 \%$ y un $25,4 \%$ de los futbolistas, respectivamente. Respecto a la gravedad, las lesiones leves son las más numerosas (con 44 casos), seguidas por lesiones moderadas (38 casos); las lesiones graves son más infrecuentes (11), afectando solo a un 18,1\% de los jugadores, y excepcionales las lesiones muy graves (6), afectando únicamente al $7 \%$ de los jugadores.

$\mathrm{El}$ análisis de correlaciones realizado indica que las lesiones muy graves ( $(\mathrm{r}=0,303$; ád»0.01) y las moderadas $(\mathrm{r}=0,258$; ád» 0.05$)$ se asocian ligeramente con las lesiones óseas. Las lesiones musculares se asocian a lesiones moderadas de un modo sensiblemente mas elevado $(\mathrm{r}=0,449$; ád»0.01), y a lesiones graves, pero con menor intensidad $(\mathrm{r}=0,331 ;$ ád»0.01). Lo más relevante es que las lesiones musculares suelen ser con lesiones moderadas, aunque también se asocian en menor medida con lesiones graves. Por último, las lesiones óseas implican lesiones que no son leves, asociándose a lesiones de mayor gravedad, siendo el único tipo de lesión que correlaciona con las lesiones muy graves.

Por otro lado, en la tabla 4, se puede observar que las medias de lesión en entrenamientos y en partidos son casi iguales en el caso del 


\begin{tabular}{|c|c|c|c|c|}
\hline \multicolumn{2}{|c|}{ Criterios de análisis } & $\begin{array}{c}\mathrm{N}^{\circ} \mathrm{de} \\
\text { lesiones }\end{array}$ & $\begin{array}{l}\mathrm{N}^{\mathrm{o}} \text { jugadores que sufren } \\
\text { lesión }\end{array}$ & Porcentaje \\
\hline \multirow{8}{*}{$\begin{array}{l}\text { LUGAR DE } \\
\text { OCURRENCIA DE LA } \\
\text { LESION }\end{array}$} & \multirow{5}{*}{$\begin{array}{l}\text { Lesiones en los entren. } \\
\mathrm{N}=68\end{array}$} & 0 & 23 & 33,3 \\
\hline & & 1 & 28 & 40,6 \\
\hline & & 2 & 15 & 21,7 \\
\hline & & 3 & 2 & 2,9 \\
\hline & & 4 & 1 & 1,4 \\
\hline & \multirow{3}{*}{$\begin{array}{l}\text { Lesiones en los partidos } \\
\mathrm{N}=33\end{array}$} & 0 & 39 & 56,5 \\
\hline & & 1 & 27 & 39,1 \\
\hline & & 2 & 3 & 4,3 \\
\hline \multirow{14}{*}{ TIPO DE LESION } & \multirow{4}{*}{$\begin{array}{l}\text { Muscular } \\
\mathrm{N}=55\end{array}$} & 0 & 32 & 45,1 \\
\hline & & 1 & 26 & 36,6 \\
\hline & & 2 & 10 & 14,1 \\
\hline & & 3 & 3 & 4,2 \\
\hline & \multirow{3}{*}{$\begin{array}{l}\text { Contusiones } \\
\mathrm{N}=11\end{array}$} & 0 & 61 & 85,9 \\
\hline & & 1 & 9 & 12,7 \\
\hline & & 2 & 1 & 1,4 \\
\hline & \multirow{2}{*}{$\begin{array}{l}\text { Esquinces } \\
\mathrm{N}=18\end{array}$} & 0 & 53 & 74,6 \\
\hline & & 1 & 18 & 25,4 \\
\hline & \multirow{3}{*}{$\begin{array}{l}\text { Tendinitis } \\
\mathrm{N}=9\end{array}$} & 0 & 63 & 88,7 \\
\hline & & 1 & 4 & 9,9 \\
\hline & & 2 & 1 & 1,4 \\
\hline & Oseas & 0 & 61 & 85,9 \\
\hline & $\mathrm{N}=10$ & 1 & 10 & 14,1 \\
\hline \multirow{15}{*}{$\begin{array}{l}\text { GRAVEDAD DE LA } \\
\text { LESIÓN }\end{array}$} & \multirow{4}{*}{$\begin{array}{l}\text { Leve } \\
\mathrm{N}=44\end{array}$} & 0 & 38 & 53,5 \\
\hline & & 1 & 25 & 35,2 \\
\hline & & 2 & 5 & 7,0 \\
\hline & & 3 & 3 & 4,2 \\
\hline & \multirow{4}{*}{$\begin{array}{l}\text { Moderada } \\
\mathrm{N}=38\end{array}$} & 0 & 42 & 59,2 \\
\hline & & 1 & 21 & 29,6 \\
\hline & & 2 & 7 & 9,9 \\
\hline & & 3 & 14 & 1,4 \\
\hline & \multirow{4}{*}{$\begin{array}{l}\text { Graves } \\
\mathrm{N}=16\end{array}$} & 0 & 58 & 81,7 \\
\hline & & 1 & 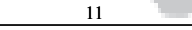 & 15,3 \\
\hline & & 2 & 1 & 1,4 \\
\hline & & 3 & 1 & 1,4 \\
\hline & \multirow{3}{*}{$\begin{array}{l}\text { Muy Graves } \\
\mathrm{N}=6\end{array}$} & 0 & 66 & 93,0 \\
\hline & & 1 & 4 & 5,6 \\
\hline & & 2 & 1 & 1,4 \\
\hline
\end{tabular}

Ranero CF; sin embargo, la media de lesiones es mucho mayor en entrenamientos que en partidos, en el caso del Real Murcia. Además, en entrenamientos la media de lesiones es muy superior en el Real Murcia respecto al Ranero, pero sucede al contrario con la media de lesiones en partidos, la del Ranero duplica a la del Real Murcia. Por lo tanto se puede decir que los jugadores del Real Murcia se lesionan más en los entrenamientos que en los partidos, pero no sucede así con los jugadores del Ranero, que se lesionan indistintamente en uno u otro contexto de práctica. Respecto al tipo de lesión, los datos son muy similares destacando el mayor número de lesiones musculares en ambos. Se observa una mayor media de lesión por contusión y por tendinitis en el Real Murcia, y por lesión ósea en el Ranero. El número de partidos perdidos por lesión también resulta muy similar en ambos clubes.

En la tabla 5, se indican los datos de aquellas variables que sus diferencias entre las medias de uno y otro club han resultado significativas estadísticamente. Así, respecto a los factores deportivos, los datos indican que los jugadores del Real Murcia entrenan unos minutos más al

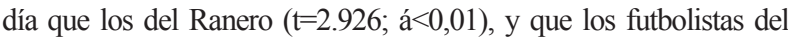
Ranero juegan más partidos que los del Real Murcia, unos seis partidos más como término medio ( $t=2.816 ; a ́<0,01)$. Respecto a los factores relacionados con las lesiones, los datos indican que los jugadores del Ranero se lesionan más en los partidos $(t=-2,465 ;$ á $<0,05)$, sin embargo, las lesiones en los entrenamientos fueron más frecuentes en el Real Murcia $(\mathrm{t}=2,942 ; \mathrm{a}<0,01)$.

\section{Discusión}

El estudio de los factores que influyen en las lesiones de los futbolistas es un tema de total y actual interés, tanto en la práctica profesional como en el fútbol de formación, que intenta establecer las bases científicas que proporcionen soluciones de carácter preventivo. Los resultados encontrados en los estudios en que se han relacionado estas variables, tanto en el ámbito profesional como formativo, nos indican que la causalidad de la lesión debe ser entendida de forma multifactorial, teniendo en cuenta, tanto factores deportivos (Emery et al., 2005; Hawkins et al., 2001; Junge et al., 2004; Kontos, 2000; Olmedilla et al., 2006), como físicos o fisiológicos (Arnason et al., 1996; Arnason et al., 2004; Hawkins y
Fuller, 1999) y psicológicos (Díaz, Buceta y Bueno, 2004; Olmedilla, Andreu y Blas, 2005).

En el presente trabajo el objetivo ha consistido en describir las lesiones sufridas, atendiendo al momento de producirse la lesión, al número de lesiones, al tipo de lesión y a la gravedad de la misma, por los futbolistas de los clubes Real Murcia y Ranero CF, y establecer si existen diferencias entre ellos.

Los resultados muestran que, agrupadas las lesiones de los jugadores de ambos clubes, las lesiones producidas durante los entrenamientos duplican a las producidas en partidos, en contra de los resultados hallados por Emery et al. (2005) y por Faude et al. (2005) en sus trabajos, en los que la mayoría de las lesiones se producían en los partidos. Este hecho puede resultar mucho más importante en el club Real Murcia, cuyos jugadores se lesionan mucho más en los entrenamientos (una media de 1,30 lesiones por jugador), que en los partidos (,30 lesiones por jugador). Algunos autores (Faude et al., 2005; Nielsen e Yde, 1989) hacen especial hincapié en alertar sobre los métodos de entrenamiento empleados, ya que excesivas cargas de trabajo podrían aumentar el riesgo de lesión. Otros (Giofsidou y Malliou, 2006; Malliou et al., 2004), indican que la ausencia de ejercicios específicos, como por ejemplo de equilibrio propioceptivo que se han mostrado útiles en la prevención de lesiones, podrían explicar parte de este hecho. Al comparar los datos entre ambos clubes, se debe de tener en cuenta que los futbolistas del Real Murcia tienen alrededor de dos días más de entrenamiento a la semana, como media, que los jugadores del Ranero, lo que también podría explicar el mayor número de lesiones de aquellos respecto a estos. Y otro dato a considerar es que los futbolistas del Ranero juegan más partidos que los del Real Murcia, unos seis partidos más como término medio; esto probablemente explique el mayor número de lesiones de los jugadores del Ranero en los partidos. En suma, parece que las diferencias encontradas entre los clubes en cuanto al lugar de ocurrencia de la lesión (en los partidos o en los entrenamientos), se deben a un aumento en los días de entrenamiento y un mayor número de partidos jugados, respectivamente.

Por otra parte las lesiones más frecuentes encontradas en este estudio son musculares y de carácter leve o moderado. Datos que van en la línea de los resultados hallados por otros trabajos (ver la revisión de Junge y Dvorak, 2004). Aunque los datos no son significativos estadísticamente, es interesante observar que los jugadores del Real Murcia tienen una media superior de lesiones leves, moderadas y graves respecto a los jugadores del Ranero; sin embargo, éstos superan, casi duplican la media de lesiones sufridas de carácter muy grave, a los jugadores del Real Murcia. Quizá, al ser una muestra no muy grande, un número pequeño de estas lesiones pueden explicar estas diferencias.

Si se observa la edad media de los jugadores de cada club $(15,31$ para el Ranero, y 19,45 para el Real Murcia), se puede ver que hay una diferencia de 4 años, lo que en estas categorías puede ser determinante, tanto en los tiempos de duración de los entrenamientos y partidos, como en la metodología de entrenamiento o en la intensidad de juego de los partidos. En este sentido, algunos estudios (Emery et al., 2005; Majewski etal., 2006) indican la influencia de la edad, y por lo tanto de la categoría deportiva de competición, en la vulnerabilidad a la lesión de los jugadores. Así, Olmedilla et al. (2006) indican que los futbolistas de categoría cadete se lesionan con mayor frecuencia que los jugadores de categorías inferiores, concretamente infantil y alevín; aunque es necesario tener en cuenta que al aumentar de categoría, se aumenta el tiempo de exposición al riesgo de lesionarse. Sin embargo, los datos del presente trabajo no indican diferencias significativas en la media de lesión de los jugadores del Real Murcia, respecto a los del Ranero, aunque la de aquellos sea superior $(1,70)$ a la de éstos $(1,29)$. Otra limitación del estudio es que, de las categorías estudiadas (cadete, juvenil y $3^{\text {a }}$ división), la muestra solo tiene representación de ambos clubes en la categoría juvenil, estando la cadete formada exclusivamente por jugadores del 


\begin{tabular}{|l|c|c|c|c|c|c|}
\hline \multicolumn{7}{|c|}{ Tabla 4. Comparación de los datos de lesiones entre el Ranero CF y el R. Murcia SAD } \\
\hline & \multicolumn{7}{|c|}{ RANERO CF } & \multicolumn{3}{c|}{ REAL MURCIA SAD } \\
\hline & $\mathrm{N}$ & Media & DT & N & Media & DT \\
\hline Lugar ocurrencia lesión & & & & & & \\
\hline - Lesión en entrenamiento & 36 &, 69 &, 70 & 33 & 1,30 &, 98 \\
\hline - Lesión en partido & 36 &, 63 &, 54 & 33 &, 30 &, 58 \\
\hline Tipo de lesión & & & & & & \\
\hline -Musculares & 38 &, 68 &, 73 & 33 &, 87 &, 96 \\
\hline - Contusiones & 38 &, 05 &, 32 & 33 &, 27 &, 45 \\
\hline - Esguinces & 38 &, 28 &, 45 & 33 &, 21 &, 41 \\
\hline - Tendinitis & 38 &, 07 &, 27 & 33 &, 18 &, 46 \\
\hline - Lesión ósea & 38 &, 18 &, 39 & 33 &, 09 &, 29 \\
\hline Gravedad de la lesión & & & & & & \\
\hline - Lesión leve & 38 &, 52 &, 60 & 33 &, 72 &, 97 \\
\hline - Lesión moderada & 38 &, 44 &, 64 & 33 &, 63 &, 82 \\
\hline - Lesión grave & 38 &, 18 &, 56 & 33 &, 27 &, 51 \\
\hline - Lesión muy grave & 38 &, 10 &, 38 & 33 &, 06 &, 24 \\
\hline$N^{\circ}$ partidos perdidos por lesión & 38 & 3,50 & 5,17 & 33 & 3,69 & 4,26 \\
\hline
\end{tabular}

Ranero, y la $3^{a}$ división por, exclusivamente, jugadores del real Murcia. Sería interesante para futuros trabajos, homogeneizar estos aspectos para, de alguna forma, tener un mayor control de las variables relativas a entrenamientos y partidos.

En definitiva, se puede afirmar, a partir de los datos de la muestra estudiada, que las lesiones producidas durante los entrenamientos duplican a las producidas en partidos, lo que debería ser un indicador de alerta sobre el tipo de entrenamiento que se realiza en estas categorías (sobre todo en el club Real Murcia); aspecto que toma mayor relevancia al conocer que las lesiones más frecuentes son de tipo muscular, lo que también es un indicador sobre la relación demanda-capacidad para cada una de las categorías, o lo que es igual para cada franja de edad en la escala evolutiva de los futbolistas.

\begin{tabular}{|l|l|c|c|c|c|c|c|c|}
\hline \multicolumn{8}{|c|}{ Tabla 5. Diferencias de medias en las variables objeto de estudio por clubes } \\
\hline & CLUB & N & MEDIA & D. T. & $\begin{array}{c}\text { Error típico } \\
\text { de la media }\end{array}$ & $\mathrm{T}$ & $\mathrm{gl}$ & $\begin{array}{c}\text { Sig } \\
\text { (bilateral) }\end{array}$ \\
\hline \multirow{2}{*}{$\begin{array}{l}\text { Entreno } \\
\text { semanal (días) }\end{array}$} & R. Murcia & 33 & 4,69 &, 58 &, 10192 & 17,408 & 69 & 0.000 \\
\hline & Ranero CF & 38 & 2,97 &, 16 &, 02632 & & & \\
\hline \multirow{2}{*}{$\begin{array}{l}\text { Lesión } \\
\text { entrenamiento }\end{array}$} & R. Murcia & 33 & 1,30 &, 98 &, 17125 & 2,924 & $57,8^{*}$ & 0.005 \\
\cline { 2 - 10 } & Ranero CF & 36 &, 69 &, 70 &, 11832 & & & \\
\hline \multirow{2}{*}{ Lesión partidos } & R. Murcia & 33 &, 30 &, 58 &, 10192 & $-2,465$ & $65,24 *$ & 0.016 \\
\cline { 2 - 10 } & Ranero CF & 36 &, 63 &, 54 &, 09044 & & & \\
\hline \multirow{2}{*}{$\begin{array}{l}\text { Entreno día } \\
\text { (min.) }\end{array}$} & R. Murcia & 33 & 3,81 &, 39 &, 06818 & 2,926 & 69 & 0.005 \\
\cline { 2 - 10 } & Ranero CF & 38 & 3,50 &, 50 &, 08220 & & & \\
\hline \multirow{2}{*}{$\begin{array}{l}\text { Partidos } \\
\text { jugados }\end{array}$} & R. Murcia & 33 & 12,90 & 6,14 & 1,06987 & $-2,816$ & 69 & 0,006 \\
\cline { 2 - 9 } & Ranero CF & 38 & 18,54 & 9,71 & 1,69172 & & & \\
\hline
\end{tabular}

\section{Limitaciones del estudio}

Aunque en líneas generales, los resultados del presente trabajo muestran datos muy interesantes, éstos deben de tomarse con cautela, debido fundamentalmente a limitaciones del estudio. Quizá las más relevantes sean referidas a la muestra y el procedimiento de registro de las lesiones. Respecto a la muestra, dos aspectos podrían ser mejorados: por un lado, aumentar el número muestral, y por otro, homogeneizar la edad o el nivel competitivo de los futbolistas. Respecto al registro de lesiones, aunque en otros trabajos se han realizado de forma similar (Abenza, Olmedilla, Ortega y Esparza, en prensa), puede ser aconsejable disponer de especialistas (fisioterapeutas) que registren las lesiones en el momento de producirse, en clasificaciones puramente médicas. Además, y aunque en otros trabajos se ha mostrado útil, una toma de datos de lesiones prospectiva podría resultar de mayor fiabilidad(Ortín, 2009).

\section{Agradecimientos}

En parte, este trabajo se ha realizado gracias a la ayuda 05691/PPC/ 07 (Fundación Séneca, Agencia Regional de Ciencia y Tecnología de Murcia) y a la ayuda 2008/09RM de la Cátedra Real Madrid y Universidad Europea de Madrid

\section{Referencias bibliográficas}

Arnason, A., Gudmundsson, A., Dahl, H.A. y Johansson, E. (1996). Soccer injuries in Iceland Scandinavian Journal of Medicine and Science in Sports, 6(1), 40-45.
Arnason, A., Sigurdsson, S.B., Gudmundsson, A., Holme, I., Engebretsen, L. y Bahr, R. (2004). Physical fitness, injuries, and team performance in soccer. Medicine and Science in Sport and Exercise, 36(2), 1459-1465.

Barcala, R.J., Palacios, J. García, J.L. y Oleagordia, A. (2007). La intervención prehospitalaria urgente en el campo de fútbol Retos. Nuevas tendencias en Educación Física, Deporte y Recreación, 12, 36-44.

Buceta, J.M. (1996). Psicología y lesiones deportivas: prevención y recuperación. Madrid: Dykinson.

Díaz, M.P., Buceta, J.M. y Bueno, A.M. (2004). Situaciones estresantes y vulnerabilidad a las lesiones deportivas: un estudio con deportistas de equipo. Revista de Psicología del Deporte, 14(1), 7-24.

Emery, C.A., Meeuwisse, W.H. y Hartmann, S.E. (2005). Evaluation of risk factors for the injury in adolescent soccer implementation and validation of an injury surveillance system. American Journal of Sports Medicine, 33(12), 1882-1891.

Faude, O., Junge, A., Kindermann, W. y Dvorak, J. (2005). Injuries in female soccer players: A prospective study in the German National League. American Journal of Sports Medicine, 33(11), 1694-1700.

Fuller, C.W., Junge, A. y Dvorak, J. (2004). An assessment of football referees decisions in incidents leading to player injuries. American Journal of Sports Medicine, 32(1), 17-22.

Gioftsidou, A. y Malliou, P. (2006). Preventing lower limb injuries in soccer players. Strength and Conditioning Journal, 28(1), 10-13.

Hägglund, M., Walden, M. y Ekstrand, J. (2003). Exposure and injury risk in Swedish elite football: a comparison between seasons 1982 and 2001. Scandinavian Journal of Medicine and Science in Sports, 13(6), 364-370.

Hawkins, R.D. y Fuller, C.W. (1998). An a examination of the frequency and severity of injuries and incidents at three levels of professional football. British Journal of Sports Medicine, 32(4), 326-332.

Hawkins, R.D. y Fuller, C.W. (1999). A prospective epidemiological study of injuries in four English professional football clubs. British Journal of Sports Medicine, 33(3), 196-203.

Hawkins, R.D., Hulse, M.A., Wilkinson, C., Hodson, A. y Gibson, M. (2001). The association football medical research programme: an audit of injuries in professional football. British Journal of Sports Medicine, 35(1), 43-47.

unge, A. y Dvorak, J. (2004). Soccer injuries: a review on incidente and prevention. Sports Medicine, 34(13), 929-938.

Junge, A., Dvorak, J. y Graf-Baumann, T. (2004). Football injuries during the World Cup 2002. American Journal of Sports Medicine, 32(10), 23-27.

Kibler, W.B. (1995). Injuries in adolescent and preadolescent soccer players. Medicine and Science in Sports and Exercise, 25(12), 1330-1332.

Kontos, A.P. (2000). The effects of perceived risk, risk-taking behaviors, and body size on injury in youth sport. Microform Publications, University of Oregon: Eugene.

Louw, Q.A., Manilall, J. y Grimmer, K.A. (2008). Epidemiology of knee injuries among adolescents: a systematic review. British Journal of Sports Medicine, 42, 2-0.

Lüthje, P., Nurmi, I., Kataja, M., et al. (1996). Epidemiology and traumatology of injuries in elite soccer: a prospective study in Finland. Scandinavian Journal of Medicine and Science in Sports, 6(3), 180-185.

Maehlum, S., Dahl, E. y Daljord, O.A. (1986). Frequency of injuries in a youth soccer tournament. Physician and Sports Medicine, 14(7), 73-80.

Majewski, M., Susanne, H. y Klaus, S. (2006). Epidemiology of athletic knee injuries: A 10-year study. Knee, 13(3), 184-188.

Malliou, P., Gioftsidou, A., Pafis, G., Beneka, A. y Godolias, G. (2004). Proprioceptive training (balance exercises) reduces lower extremity injuries in young soccer players. Journal of Back and Musculoskeletal Rehabilitation, 17, 101-104.

Morgan, B.E. y Oberlander, M.A. (2001). An a examination of injuries in major league soccer: the inaugural season. American Journal of Sports Medicine, 29(4), 426430.

Nicholl, J.P., Coleman, P. y Williams, B.T. (1995). The epidemiology of sports and exercise related injury in the United Kingdom. British Journal of Sports Medicine, 29(4), 232-238.

Nielsen, A.B. y Yde, J. (1989). Epidemiology and traumatology of injuries in soccer. American Journal of Sports Medicine, 17(6), 803-807.

Olmedilla, A., Andreu, M.D. y Blas, A. (2005). Variables psicológicas, categorías deportivas y lesiones en futbolistas jóvenes: un estudio correlacional. Analise Psicologica, 4(XXIII), 449-459.

Olmedilla, A., Andreu, M.D., Abenza, L., Ortín, F.J. y Blas, A. (2006). Lesiones y factores deportivos en futbolistas jóvenes. Cultura, Ciencia y Deporte, 5(2), 59-66.

Olmedilla, A., García-Montalvo, C. y Martínez-Sánchez, F. (2006). Factores psicológicos y vulnerabilidad a las lesiones deportivas: un estudio en futbolistas. Revista de Psicología del Deporte, 15(1), 37-52.

Olmedilla, A., Ortega, E. y Abenza, L. (2007). Percepción de los futbolistas juveniles e influencia del trabajo psicológico en la relación entre variables psicológicas y lesiones. Cuadernos de Psicología del Deporte, 7(2), 75-87.

Abenza, L., Olmedilla, A., Ortega, E. y Esparza, F. (en prensa). Lesiones y factores psicológicos en futbolistas juveniles. Archivos de Medicina del Deporte.

Ortín, F.J. (2009). Factores psicológicos y socio-deportivos y lesiones en jugadores de fútbol semiprofesionales y profesionales. Tesis doctoral no publicada. Universidad de Murcia.

Östenberg, A. y Roos, H. (2000). Injury risk factors in female European football: a prospective study of 123 players during one season. Scandinavian Journal of Medicine and Science in Sports, 10(5), 279-285.

Schmitd-Olsen, S., Buenemann, L.K.H., Lade, V. y Brassoe, J.O.K. (1985). Soccer injuries of youth. British Journal of Sports Medicine, 19(3), 161-164.

Schmitd-Olsen, S., Jorgensen, U., Kalund, S. y Sorensen, J. (1991). Injuries among young soccer players. American Journal of Sports Medicine, 19(3), 273-275.

SETRADE (2007). Nota informativa de la Asociación Española de Médicos de Equipos de Fútbol (AEMEF) ante el aumento de lesiones. Obtenida en http:// www.setrade.org/publica/noticias/ficha.php?id=22

Söderman, K., Pietilä, T., Alfredson, H. y Werner, S. (2002). Anterior cruciate ligament injuries in young females playing soccer at senior levels. Scandinavian Journal of Medicine and Science in Sports, 12, 65-68. 